\section{Adesão das revistas brasileiras de saúde às práticas de ciência aberta pelos critérios SciELO Brasil}

Resumo: As práticas de ciência aberta buscam ampliar a visibilidade e democratizar o acesso e uso do conhecimento científico. Esta pesquisa busca analisar a adesão das revistas brasileiras de ciências da saúde às práticas de ciência aberta estimuladas pelos critérios SciELO Brasil, publicados em maio de 2020. Os resultados demonstram a adesão satisfatória a algumas práticas e a baixa adesão a outras. Conclui que devem ser impulsionados os processos de abertura da avaliação por pares, o compartilhamento de dados, a publicação de preprints e o uso da taxonomia CRediT para o esclarecimento da contribuição dos autores.

Palavras-chave: Revistas Científicas. Ciências da Saúde. Brasil.

Adherence of Brazilian health journals to the open science practices from the SciELO Brazil criteria

Abstract: Open science practices seek to expand visibility and democratize the access and use of scientific knowledge. This research intends to analyze the adherence of Brazilian health science journals to the open science practices encouraged by the SciELO Brasil criteria, published in May 2020. The results show satisfactory adherence to some practices and low adherence to others. It concludes that the processes of open peer review, data sharing, the publication of preprints, and the use of the CRediT taxonomy to clarify the authors' contribution should be promoted.

Keywords: Scientific Journals. Health Sciences. Brazil.

Adhesión de las revistas brasileñas de salud a las prácticas de ciencia abierta por los criterios SciELO Brasil

Resumen: Las prácticas de ciencia abierta buscan ampliar la visibilidad y democratizar el acceso y uso del conocimiento científico. Esta investigación busca analizar la adhesión de las revistas brasileñas de ciencias de la salud a las prácticas de ciencia abierta estimuladas por los criterios SciELO Brasil, publicados en mayo de 2020. Los resultados demuestran una adhesión satisfactoria a algunas prácticas y una baja adhesión a otras. Concluye que deben ser impulsados los procesos para abrir la revisión por pares, fomentar el intercambio de datos, fomentar la publicación de preprints y el uso de la taxonomía CRediT para aclarar la contribución de los autores.

Palavras Clave: Revistas Científicas. Ciencias de la Salud. Brasil.

\author{
LYVIA ROCHA DE \\ JESUS ARAUJO \\ Rio de Janeiro, RJ, \\ Brasil
}

ELOÍSA PRÍNCIPE

Rio de Janeiro, RJ, Brasil 


\section{Introdução}

Desde a última década do século XX movimentos como o Open Access (acesso aberto) e o Open Archives (arquivos abertos) vieram derrubando as barreiras que dificultavam o acesso às informações científicas e documentárias que começavam a se proliferar nos espaços digitais.

No caso das publicações científicas, o movimento de acesso aberto abriu caminho para outros movimentos relacionados, como a ciência aberta que, além de garantir o acesso gratuito ao que vinha sendo publicado nas revistas científicas, pretende alcançar a abertura de todos os seus processos, dados e ferramentas.

Dentre os atores envolvidos na adoção e na difusão das práticas de ciência aberta, Sales e Shintaku (2019) destacam as editoras públicas e privadas de publicações acadêmicas e periódicos científicos, desafiadas a renovar suas políticas e critérios para adequarem-se às especificidades dispostas sob o grande guarda-chuva do movimento.

O item 5.2.10 da mais recente atualização dos critérios, políticas e procedimentos para a admissão e a permanência de periódicos científicos na Coleção SciELO Brasil $(2020)^{1}$ convida as revistas brasileiras a atualizarem seus modelos e processos de publicação para se adaptarem progressivamente aos novos moldes da ciência aberta.

Para tanto, os editores científicos devem: a) liberar o acesso integral de suas publicações, aderindo à cobrança de taxas de submissão, avaliação e/ou publicação dos autores se assim desejarem; b) encorajar a publicação de preprints como pontapé inicial para o fluxo de comunicação das pesquisas c) encorajar o compartilhamento dos dados brutos da pesquisa em repositórios de dados e, quando for o caso, o referenciamento de dados reusados; d) possibilitar a abertura do processo de revisão por pares; e) estabelecer uma licença adequada para acesso e uso dos artigos publicados e de seus metadados; f) desenvolver plano de marketing e divulgação científica; e g) promover a interoperabilidade (SCiELO, 2020).

Tomando por base os pontos "a, b, c, d, e" citados e os itens 5.2.6.4.1 - créditos aos autores e 5.2.8.3 - identificação ORCID dos critérios SciELO, esta pesquisa objetiva analisar a adesão das revistas brasileiras de ciências de saúde indexadas na Coleção SciELO Brasil às práticas da Ciência Aberta estimuladas pela rede.

\section{$2 \quad$ Metodologia}

${ }^{1}$ https://wp.scielo.org/wp-content/uploads/20200500-Criterios-SciELO-Brasil.pdf. Acesso em: 2 set. 2021. 
A pesquisa utiliza abordagem bibliográfica e exploratória.

Em um primeiro momento, foi realizado um levantamento das revistas brasileiras indexadas na SCIELO até 31 de maio de 2021, cujos registros foram exportados no formato de planilha Excel e contabilizados por áreas do conhecimento. Optou-se por utilizar como foco desta pesquisa as Ciências da Saúde, considerando o forte engajamento desta área nas iniciativas de abertura dos processos científicos.

Coletados os dados nos respectivos sites de cada revista, foi empregada análise quantitativa para a verificação das licenças Creative Commons adotadas, da abertura do processo de revisão por pares, do encorajamento ao compartilhamento de dados de pesquisa e a publicação de artigos de dados (data papers), da publicação e/ou encorajamento da publicação de preprints, da adoção da taxonomia CRediT para declaração de contribuição da autoria e do uso de identificador persistente para autores e publicações.

\section{Resultados e discussão}

O levantamento na Coleção SciELO Brasil resultou num total de 303 revistas brasileiras ativas categorizadas em uma ou mais de $u \mathrm{ma}^{2}$ das oito grandes áreas do conhecimento da Tabela de Áreas do Conhecimento do Conselho Nacional de Desenvolvimento Científico e Tecnológico - CNPq.

De acordo com o recorte selecionado para esta pesquisa, foram recuperadas 98 revistas classificadas na grande área das Ciências da Saúde, 78 delas categorizadas exclusivamente nessa área e 20 outros títulos também classificados em outras grandes áreas - 12 nas Ciências Humanas, quatro nas Ciências Biológicas e quatro distribuídas simultaneamente em três ou mais áreas (Agronomia, Ciências Exatas e da Terra, Ciências Sociais Aplicadas e/ou Engenharias).

Todas as revistas consultadas fornecem acesso aberto integral aos seus conteúdos, através da via dourada, com acesso e uso irrestritos. Destas, 33 citaram estar em conformidade com os princípios defendidos pela Declaração de Acesso Aberto de Budapeste (BOAI) ${ }^{3}$.

Cabe ressaltar que o acesso aberto gratuito ao conteúdo dessas revistas não significa que a publicação em seus volumes seja livre de custos para os autores que

\footnotetext{
${ }^{2}$ As revistas indexadas na SCIELO podem ser categorizadas em mais de uma área do conhecimento simultaneamente.

${ }^{3}$ http://www.budapestopenaccessinitiative.org/reade. Acesso em: 15 jun. 2021.
} 
desejam publicar. Das revistas consultadas, 54\% não cobram nenhuma taxa de submissão, avaliação e/ou processamento de artigos, 38\% das revistas indicaram a cobrança de taxas e fornecem os valores cobrados nas instruções de submissão para os autores e em $8 \%$ das revistas não foram localizadas informações que indicassem necessidade de pagamento ou isenção de taxa.

Nesse contexto, verifica-se que o baixo investimento de recursos públicos em educação e pesquisa atinge diretamente as revistas científicas brasileiras, fazendo com que algumas editoras tenham a necessidade de cobrar taxas aos autores que desejem publicar seus trabalhos em seus títulos, para que consigam mantê-los ativos. Dentro disso, observou-se que não há um padrão de cobrança determinado para este fim, cada revista consultada estabelece o valor e as condições específicas de suas taxas, sendo $R \$ 100,00$ o menor preço e $\mathrm{R} \$ 3.300,00$ o maior.

Outro ponto diretamente relacionado à abertura das publicações destas revistas são as licenças creative commons, criadas em 2001 por Lawrence Lessig, Hal Abelson e Eric Eldred para o uso legal de materiais em acesso aberto (BRANCO; BRITTO, 2013). A maioria das revistas analisadas (79) está sob a licença de tipo $C C B Y$, que permite a distribuição, adaptação ou remixagem e a criação de obras derivadas do conteúdo e, segundo as orientações da SciELO, é a licença mais adequada para publicações abertas. Outras 12 revistas estão sob o tipo $C C B Y-N C$, que também permite a adaptação, a distribuição e a derivação do conteúdo, desde que não sejam aplicados para fins comerciais, e oito estão sob o tipo mais restritivo de licenciamento: CC BY-NC-ND, que além de não permitir uso comercial proíbe quaisquer modificações à obra original.

Uma vez que os artigos submetidos à revistas científicas geralmente tenham seus direitos de autoria e distribuição atribuídos a revista, é importante que os autores conheçam bem as licenças sob as quais suas pesquisas podem ser submetidas para que conheçam as condições legais do compartilhamento do seu conteúdo e para que o licenciamento dos artigos que pretendam submeter esteja alinhado com a licença atribuída a revista.

As práticas da ciência aberta sugerem que o processo de avaliação por pares seja aberto, possibilitando um diálogo mais acessível e transparente entre autores e pareceristas. Contudo, das revistas consultadas, apenas oito indicam que utilizam ou fornecem a opção de abertura da avaliação, expondo as identidades dos autores e dos revisores quando ambos estiverem de acordo. A maior parte das revistas (85) emprega o modelo fechado de revisão por pares, 76 utilizando os sistema duplo-cego, cujas 
identidades dos autores e dos revisores são anonimizadas, e nove sob o sistema simplescego, em que a identidade dos revisores é anonimizada mas os autores são identificados 4 .

No que concerne ao compartilhamento de dados de pesquisa, nenhuma das revistas de saúde na coleção SciELO Brasil publica data papers (artigos de dados), orientados a apresentação dos metadados que descrevem a estrutura, o formato, os métodos utilizados na coleta, organização e processamento dos dados de pesquisa. 33 delas encorajaram o depósito dos dados de pesquisa em repositórios específicos, dentre os quais indicam: SciELO Data ${ }^{5}$; Mendeley data ${ }^{6}$; Dryad $^{7} ;$ Figshare $^{8}$; Zenodo ${ }^{9}$; Repositório de Dados Científicos da Universidade de São Paulo (USP) ${ }^{10}$ e o GenBank ${ }^{11}$.

Apesar de haver a possibilidade do depósito de dados de pesquisa em repositórios de dados ou repositórios institucionais ou temáticos e isso representar um grande avanço para o movimento da ciência aberta, acredita-se que a publicação de data papers poderia colaborar ainda mais com a visibilidade e acesso aos dados de pesquisa, uma vez que os data papers podem ser indexados e recuperados em bases de dados tradicionais, como é o caso da Web of Science (WoS) e da Scopus. Essa nova forma de publicação torna um conjunto de dados reutilizável e reprodutível, mediante descrição aprofundada que permite sua exploração livre, através da atribuição de um identificador persistente que também o torna citável.

Todas as revistas consultadas para esta pesquisa fornecem o Digital Object Identifier (DOI) para suas publicações, mas nem todas exigem ou recomendam o uso do Open Researcher and Contributor ID (ORCID). É exigido uso do ORCID na submissão de manuscritos de 78 revistas e em uma seu uso é apenas recomendado. 19 revistas ainda não o citam em suas diretrizes.

Outro ponto analisado foi a aceitação e/ou publicação de preprints pelas revistas da área da saúde, onde verificou-se que 31 revistas aceitam trabalhos que já tenham sido publicados nesse modelo de publicação, sendo citadas como exemplos as seguintes

\footnotetext{
${ }^{4}$ Cinco revistas ficaram de fora desta contagem pois não foram localizadas informações que esclarecessem o sistema e o modelo empregados.

${ }^{5}$ https://data.scielo.org/dataverse/scielodata. Acesso em: 15 jun. 2021.

${ }^{6} \mathrm{https}: / /$ data.mendeley.com/. Acesso em: 15 jun. 2021.

7 https://datadryad.org/stash. Acesso em: 15 jun. 2021.

8 https://figshare.com/. Acesso em: 15 jun. 2021.

9 https://zenodo.org/. Acesso em: 15 jun. 2021.

$10 \mathrm{https}: / /$ uspdigital.usp.br/repositorio/. Acesso em: 15 jun. 2021.

11 https://ftp.ncbi.nlm.nih.gov/genbank/. Acesso em 15 jun. 2021.
} 
plataformas nas quais os preprints possam ser depositados: SciELO preprints ${ }^{12}$; $\operatorname{ArXiv}^{13}$; BioRxiv ${ }^{14}$.

A publicação de preprints nas ciências da saúde disponibiliza resultados preliminares, iniciais ou intermediários de pesquisas ainda não concluídas, que podem ser de grande contribuição para outros pesquisadores que estejam desenvolvendo pesquisas similares.

Por fim, espera-se que os critérios de atribuição de autoria se dêem através do registro explícito da colaboração de cada autor e co-autor dos manuscritos submetidos à publicação. Para que isso seja feito é sugerida a adoção da taxonomia Contributor Roles Taxonomy (CRediT) ${ }^{15}$ ou sistemas equivalentes, como as normas do International Committee of Medical Journal Editors (ICMJE) ${ }^{16}$. Apenas $7 \%$ das revistas consultadas apontam para o uso da taxonomia CRediT.

Em geral, é comum que as revistas das ciências da saúde sigam as orientações do ICMJE no que se refere à estruturação de seus conteúdos, acredita-se que este fato influencia no baixo uso da taxonomia CRediT.

\section{Considerações finais}

Esta pesquisa buscou analisar a adesão das revistas brasileiras das Ciências da Saúde indexadas na Coleção SciELO Brasil às práticas de ciência aberta.

A análise dos dados coletados demonstrou que todas as revistas seguem a via dourada de acesso aberto, possibilitando acesso e uso gratuito dos seus conteúdos e atendendo adequadamente aos critérios SciELO.

Quanto à cobrança de taxa aos autores, os critérios SciELO deixam a cargo das revistas cobrá-las ou não. Nesse quesito, $54 \%$ das revistas consultadas não cobram aos autores nenhuma taxa de submissão, avaliação ou processamento de artigos por serem financiadas por associações e/ou instituições públicas, o que torna seu processo de publicação mais acessível.

No que tange à permissão de uso e aplicação dos conteúdos destas revistas, em sua grande maioria (80\%) elas estão sob a licença creative commons do tipo $C C B Y$, o

\footnotetext{
12 https://preprints.scielo.org/index.php/scielo. Acesso em: 15 jun. 2021.

13 https://arxiv.org/. Acesso em: 15 jun. 2021.

${ }^{14}$ https://www.biorxiv.org/. Acesso em: 15 jun. 2021.

15 https://casrai.org/credit/. Acesso em: 15 jun. 2021.

16 http://www.icmje.org/recommendations/translations/portugese2014.pdf. Acesso em: 15 jun. 2021.
} 
modelo de distribuição e permissividade mais abrangente de conteúdo previsto pela organização e o mais recomendado pelos critérios SciELO.

Observou-se que o fornecimento de identificadores persistentes também vem sendo bem empregado pelas revistas consultadas, em que $100 \%$ fornece um DOI para seus artigos e $80 \%$ exige ou recomenda o ORCID dos autores na etapa de submissão.

Mas, apesar da adaptação das revistas de saúde às mudanças requeridas pelos critérios de admissão e permanência de revistas na coleção SciELO Brasil estarem bem encaminhadas, ainda existem práticas que precisam ser melhor exploradas pelos editores científicos. Dentre elas, destacam-se: a abertura do processo de avaliação por pares, cujos resultados demonstraram que apenas $8 \%$ das revistas adotam ou oferecem esta opção; 0 compartilhamento de dados de pesquisa em repositórios, encorajado por $1 / 3$ das revistas consultadas; a publicação de preprints, aceita por 31\% das revistas; e o uso da taxonomia CRediT, apontado em apenas 7\% das revistas, enquanto 39\% delas indicaram o emprego das normas de contribuição de autoria do ICMJE. Revela-se, assim, a necessidade de mais ações de conscientização direta e objetiva das práticas da ciência aberta junto aos pesquisadores, editores, órgãos de políticas públicas e sociedade como um todo, de forma a se obter um ambiente mais inclusivo, colaborativo e transparente na atividade científica.

\section{Referências}

BRANCO, Sérgio; BRITTO, Walter. O que é Creative Commons? Novos modelos de direito autoral em um mundo mais criativo. Rio de Janeiro: Editora FGV, 2013. Disponível em:

https://bibliotecadigital.fgv.br/dspace/bitstream/handle/10438/11461/0\%20que\%20\%C3\% A9\%20Creative\%20Commons.pdf. Acesso em: 15 jun. 2021.

SALES, Luana Farias; SHINTAKU, Milton. A ciência aberta e a necessidade de novos modelos de publicação. In: SHINTAKU, Milton; SALES, Luana Farias (org.). Ciência aberta para editores científicos. Botucatu: ABEC, 2019. p. 11-14. Disponível em: https://www.abecbrasil.org.br/arquivos/Ciencia aberta editores cientificos Ebook.pdf. Acesso em: 16 jun. 2021.

SCIENTIFIC ELECTRONIC LIBRARY ONLINE. Critérios, políticas e procedimentos para a admissão e a permanência de periódicos científicos na Coleção SciELO Brasil. 2020. Disponível em: https://wp.scielo.org/wp-content/uploads/20200500-CriteriosSciELO-Brasil.pdf. Acesso em: 15 jun. 2021. 
Lyvia Rocha de Jesus Araujo

Rio de Janeiro, RJ, Brasil

Programa de Pós-graduação em Ciência da Informação, convênio Ibict/UFRJ

Mestranda em Ciência da Informação no Programa de Pós-Graduação em Ciência da Informação (PPGCI IBICT/UFRJ) e bacharel em Biblioteconomia (2021) pela Universidade Federal do Estado do Rio de Janeiro (UNIRIO)

Contribuição de autoria: Conceituação, Metodologia, Curadoria de Dados, Análise formal, Investigação, Visualização, Escrita - Primeira Redação

ORCID ID: https://orcid.org/0000-0002-4347-106X

Lattes: http://lattes.cnpq.br/0001022583579673.

E-mail: araujolyvia@ufrj.br

\section{Eloísa Príncipe}

Rio de Janeiro, RJ, Brasil

Programa de Pós-graduação em Ciência da Informação, convênio Ibict/UFRJ

Doutora (2005) e Mestre em Ciência da Informação (1989) pela Universidade Federal do Rio de Janeiro (UFRJ), especialista em Documentação Científica (1979) e bacharel (1978) em Biblioteconomia e Documentação pela Universidade Federal Fluminense (UFF). Professora do Programa de Pós-Graduação em Ciência da Informação (convênio UFRJ-IBICT). Tecnologista Sênior do Instituto Brasileiro de Informação em Ciência e Tecnologia (IBICT), unidade de pesquisa do Ministério da Ciência, Tecnologia, Inovações e Comunicações (MCTI), desde 1979, onde desenvolve atividades técnicas, gerenciais e acadêmicas. É membro da Associação Brasileira de Editores Científicos (ABEC) da qual já foi membro da Diretoria, por dois mandatos, além de participar de seu Conselho Deliberativo (2018/2022, 2010/2014, 1997/2001), e colaborar com a Associação de forma intensa, em palestras e treinamentos de editoração científica. Participa do Grupo de Pesquisa - Comunicação e Divulgação Científicas (vice-líder) do IBICT, certificado pelo CNPq. Coordenadora do projeto de pesquisa sobre comunicação científica, suas práticas, padrões e tendências em seus múltiplos aspectos. Tem trabalhos publicados e participa de reuniões científicas da área. Ex-Editora Adjunta da revista Tendências da Pesquisa Brasileira em Ciência da Informação, publicada pela Associação Nacional de Pesquisa e Pós-Graduação em Ciência da Informação (ANCIB) e parecerista dos periódicos Ciência da Informação, Informação \& Informação, Liinc em Revista, RDBCl - Revista Digital de Biblioteconomia, RBBD - Revista Brasileira de Biblioteconomia e Documentação, RECIIS - Revista Eletrônica de Comunicação, Informação e Inovação em Saúde e Transinformação. Atua na área de Ciência da Informação, com ênfase nos seguintes temas: comunicação científica, ciência aberta, acesso aberto, periódicos científicos, estudos métricos da informação, tecnologias da informação e da comunicação (TIC).

Contribuição de autoria: Conceituação, Administração do projeto, Metodologia, Escrita - Revisão e Edição, Supervisão e Validação

ORCID ID: https://orcid.org/0000-0002-1540-0854

Lattes: http://lattes.cnpq.br/4433049915911546.

E-mail: principe@ibict.br 